\title{
Synthesis of Enantiopure Imidazolines through a Ritter Reaction of 2-(1-Aminoalkyl)aziridines with Nitriles
}

\author{
José M. Concellón, ${ }^{*[a]}$ Estela Riego, ${ }^{[a]}$ José Ramón Suárez, ${ }^{[a]}$ Santiago García-Granda ${ }^{[b]}$ \\ and M. Rosario Díaz ${ }^{[b]}$ \\ ${ }^{[a]}$ Departamento de Química Orgánica e Inorgánica, \\ Facultad de Química, Universidad de Oviedo, Julián \\ Clavería, 8, 33071 Oviedo, Spain. \\ ${ }^{[b]}$ Departamento de Química Física y Analítica, Facultad \\ de Química, Universidad de Oviedo, Julián Clavería, 8, \\ 33071 Oviedo, Spain. \\ Email:jmcg@sauron.quimica.uniovi.es
}

\section{GENERAL:}

All reactions were carried out under an atmosphere of dry $\mathrm{N}_{2}$ using oven-dried glassware and syringes. All reagents were purchased in the higher quality available and were used without further purification. $\mathrm{BF}_{3} \cdot \mathrm{OEt}_{2}$ and acetonitrile were distilled from $\mathrm{CaH}_{2}$ and stored over activated $4 \AA$ molecular sieves. The solvents used in column chromatography, $\mathrm{CH}_{2} \mathrm{Cl}_{2}, \mathrm{MeOH}$ were obtained from commercial suppliers and used without further distillation. TLC was performed on aluminium-backed plates coated with silica gel 60 with $\mathrm{F}_{254}$ indicator (Scharlau). Flash column chromatography was carried out on silica gel 60, 230-240 mesh. ${ }^{1} \mathrm{H}$ NMR $(200,300,400 \mathrm{MHz})$ and ${ }^{13} \mathrm{C}$ NMR $(50.5,75.5,100 \mathrm{MHz})$ spectra were measured at room temperature on a Bruker AC-200, AC-300 and AMX-400 instruments, respectively, with tetramethylsilane $\left(\delta=0.0,{ }^{1} \mathrm{H}\right.$ NMR $)$ or $\mathrm{CDCl}_{3}\left(\delta=77.00,{ }^{13} \mathrm{C} \mathrm{NMR}\right)$ as internal standard. Carbon multiplicities were assigned by DEPT techniques. Low-resolution electron impact mass spectra (EILRMS) were obtained at $70 \mathrm{eV}$ on a HP $5987 \mathrm{~A}$, and the intensities are reported as a percentage relative to the base peak after the corresponding $\mathrm{m} / \mathrm{z}$ value. High-resolution mass spectra (HRMS) were determined on a Finnigan MAT 95 spectrometer. Elemental analyses were carried out on a Perkin-Elmer 2400 and Carlo Erba 1108 microanalyzers. 


\section{SPECTROSCOPIC DATA OF COMPOUNDS 2:}

(4R,5R)-1-Benzyl-5-(benzylaminomethyl)-4-isobutyl-2-methyl-4,5-dihydro-1 $H$-imidazole (2a): Colorless oil. $[\alpha]^{25}=+15.3\left(c 0.20, \mathrm{CHCl}_{3}\right) ;{ }^{1} \mathrm{H}$ NMR $\left(300 \mathrm{MHz}, \mathrm{CDCl}_{3}\right): \delta 7.25-7.13(\mathrm{~m}, 10 \mathrm{H}), 4.38$ (s, 2 H), 3.81-3.77 (m, 1 H), 3.69 (s, 2 H), $3.16(\mathrm{dd}, J=8.6,4.3 \mathrm{~Hz}, 1 \mathrm{H}), 2.63$ (ddd, $J=16.8,12.2,4.6$ $\mathrm{Hz}, 2 \mathrm{H}), 2.03$ (s, $3 \mathrm{H}), 1.85-1.78$ (m, $1 \mathrm{H}), 1.51-1.44$ (m, $1 \mathrm{H}), 1.22$ (br s, $1 \mathrm{H}), 1.17-1.08$ (m, $1 \mathrm{H}), 0.91$ $(\mathrm{d}, J=6.0 \mathrm{~Hz}, 3 \mathrm{H}), 0.86(\mathrm{~d}, J=6.0 \mathrm{~Hz}, 3 \mathrm{H}) ;{ }^{13} \mathrm{C} \mathrm{NMR}\left(75 \mathrm{MHz}, \mathrm{CDCl}_{3}\right): \delta 164.1(\mathrm{C}), 140.0(\mathrm{C}), 136.0$ (C), 128.8, 128.2, 128.1, 127.8, $126.9(10 \times \mathrm{CH}), 66.2(\mathrm{CH}), 62.1(\mathrm{CH}), 53.7\left(\mathrm{CH}_{2}\right), 49.7\left(\mathrm{CH}_{2}\right), 48.1$ $\left(\mathrm{CH}_{2}\right), 45.8\left(\mathrm{CH}_{2}\right), 24.2(\mathrm{CH}), 22.8\left(\mathrm{CH}_{3}\right), 22.3\left(\mathrm{CH}_{3}\right), 13.5\left(\mathrm{CH}_{3}\right)$; MS (70 eV, EI) $\mathrm{m} / z(\%) 349\left[M^{+}\right.$, <1], 229 (41), 173 (68), 91 (100), 69 (5); HRMS (70 eV) calc. for $\mathrm{C}_{23} \mathrm{H}_{31} \mathrm{~N}_{3}\left[M^{+}\right]$349.5236, found 349.5236; IR (neat): 3336, 3029, 2955, 2360, 2342, 1605, 1454, $1062 \mathrm{~cm}^{-1} ; R_{f}=0.31(\mathrm{MeOH})$.Anal. Calcd for $\mathrm{C}_{23} \mathrm{H}_{31} \mathrm{~N}_{3}$ : C, 79.04; H, 8.94; N, 12.02. Found: C, 79.32; H, 9.10; N, 11.87.

(4R,5R)-1-Benzyl-5-(benzylaminomethyl)-4-isobutyl-2-ethyl-4,5-dihydro-1H-imidazole (2b): Colorless oil. $[\alpha]_{\mathrm{D}}^{25}=+16.7\left(c 0.10, \mathrm{CHCl}_{3}\right) ;{ }^{1} \mathrm{H} \mathrm{NMR}\left(300 \mathrm{MHz}, \mathrm{CDCl}_{3}\right): \delta 7.35-7.14(\mathrm{~m}, 10 \mathrm{H}), 4.36$ (AB syst., $J=16.5 \mathrm{~Hz}, 2 \mathrm{H}), 3.81-3.72(\mathrm{~m}, 1 \mathrm{H}), 3.69$ (s, $2 \mathrm{H}), 3.15-3.10(\mathrm{~m}, 1 \mathrm{H}), 2.66-2.55(\mathrm{~m}, 2 \mathrm{H})$, 2.34-2.26 (m, 2 H), 1.80-1.68 (m, 1 H), 1.53-1.43 (m, $1 \mathrm{H}), 1.21$ (t, J = 7.5 Hz, 3 H), 1.18-1.08 (m, $1 \mathrm{H})$, $0.90(\mathrm{~d}, J=2.3 \mathrm{~Hz}, 3 \mathrm{H}), 0.88(\mathrm{~d}, J=2.6 \mathrm{~Hz}, 3 \mathrm{H}) ;{ }^{13} \mathrm{C} \mathrm{NMR}\left(75 \mathrm{MHz}, \mathrm{CDCl}_{3}\right): \delta 166.1(\mathrm{C}), 140.3(\mathrm{C})$, $138.1(\mathrm{C}), 128.6,128.2,127.9,127.2,126.8(10 \times \mathrm{CH}), 65.8(\mathrm{CH}), 65.7(\mathrm{CH}), 53.8\left(\mathrm{CH}_{2}\right), 51.2\left(\mathrm{CH}_{2}\right)$, $48.2\left(\mathrm{CH}_{2}\right), 46.9\left(\mathrm{CH}_{2}\right), 24.3(\mathrm{CH}), 22.9\left(\mathrm{CH}_{3}\right), 22.7\left(\mathrm{CH}_{3}\right), 21.3\left(\mathrm{CH}_{2}\right), 11.2\left(\mathrm{CH}_{3}\right) ; \mathrm{MS}(70 \mathrm{eV}, \mathrm{EI}) \mathrm{m} / \mathrm{z}$ (\%) $363\left[M^{+}, 5\right], 244$ (30), 243 (99), 187 (51), 91 (100), 71 (39), 57 (58); HRMS (70 eV) calc. for $\mathrm{C}_{24} \mathrm{H}_{33} \mathrm{~N}_{3}\left[M^{+}\right]$363.2669, found 363.2656; IR (neat): 3312, 3063, 3028, 2953, 2360, 2342, 1615, 1454 $\mathrm{cm}^{-1} ; R_{f}=0.40(\mathrm{MeOH})$; Anal. Calcd for $\mathrm{C}_{24} \mathrm{H}_{33} \mathrm{~N}_{3}$ : C, 79.29; H, 9.15; N, 11.56. Found: C, 79.41; H, $9.06 ; \mathrm{N}, 11.71$.

(4R,5R)-1-Benzyl-5-(benzylaminomethyl)-4-isobutyl-2-isopropyl-4,5-dihydro-1H-imidazole (2c): Colorless oil. $[\alpha]_{\mathrm{D}}^{25}=-8.8\left(c 0.16, \mathrm{CHCl}_{3}\right) ;{ }^{1} \mathrm{H} \mathrm{NMR}\left(300 \mathrm{MHz}, \mathrm{CDCl}_{3}\right): \delta 7.31-7.13(\mathrm{~m}, 10 \mathrm{H}), 4.38$ (AB syst., $J=16.5 \mathrm{~Hz}, 2 \mathrm{H}), 3.79-3.72(\mathrm{~m}, 1 \mathrm{H}), 3.70$ (s, $2 \mathrm{H}), 3.14(\mathrm{dd}, J=10.5,4.6 \mathrm{~Hz}, 1 \mathrm{H}), 2.66-2.51$ $(\mathrm{m}, 3 \mathrm{H}), 1.70-1.51(\mathrm{~m}, 1 \mathrm{H}), 1.50-1.43(\mathrm{~m}, 1 \mathrm{H}), 1.26-1.07(\mathrm{~m}$, with d at 1.20, J = 6.8 Hz, $8 \mathrm{H}), 0.89(\mathrm{~d}$, $J=1.7 \mathrm{~Hz}, 3 \mathrm{H}), 0.87(\mathrm{~d}, J=1.7 \mathrm{~Hz}, 3 \mathrm{H}) ;{ }^{13} \mathrm{C} \mathrm{NMR}\left(75 \mathrm{MHz}, \mathrm{CDCl}_{3}\right): \delta 168.3(\mathrm{C}), 139.4(\mathrm{C}), 137.3$ (C), $127.6(2 \times \mathrm{CH}), 127.3(2 \times \mathrm{CH}), 126.9(2 \times \mathrm{CH}), 126.3(\mathrm{CH}), 125.9(3 \times \mathrm{CH}), 64.8(\mathrm{CH}), 64.3$ $(\mathrm{CH}), 52.9\left(\mathrm{CH}_{2}\right), 50.5\left(\mathrm{CH}_{2}\right), 47.0\left(\mathrm{CH}_{2}\right), 45.8\left(\mathrm{CH}_{2}\right), 25.3(\mathrm{CH}), 23.3(\mathrm{CH}), 22.6\left(\mathrm{CH}_{3}\right), 21.4\left(\mathrm{CH}_{3}\right)$, $20.3\left(\mathrm{CH}_{3}\right), 19.3\left(\mathrm{CH}_{3}\right)$; MS (70 eV, EI) m/z (\%) 377 [M+ 8$], 258$ (48), 257 (100), 201 (58), 91 (94), 57 (47); HRMS (70 eV) calc. for $\mathrm{C}_{25} \mathrm{H}_{35} \mathrm{~N}_{3}\left[M^{+}\right]$377.2826, found 377.2815; IR (neat): 3316, 3028, 2955 , 2927, 2868, 1605, 1454, $1364 \mathrm{~cm}^{-1} ; R_{f}=0.36(\mathrm{MeOH})$; Anal. Calcd for $\mathrm{C}_{25} \mathrm{H}_{35} \mathrm{~N}_{3}$ : C, 79.53; H, 9.34; N, 11.13. Found: C, 79.72; H, 9.53; N, 10.90 .

$(4 R, 5 R)-5$-(Allylaminomethyl)-1-benzyl-4-isobutyl-2-methyl-4,5-dihydro-1H-imidazole

(2d): Colorless oil. $[\alpha]_{\mathrm{D}}^{25}=+11.4\left(c 1.30, \mathrm{CHCl}_{3}\right) ;{ }^{1} \mathrm{H}$ NMR $\left(300 \mathrm{MHz}, \mathrm{CDCl}_{3}\right): \delta 7.37-7.17(\mathrm{~m}, 5 \mathrm{H}), 5.85-$ $5.71(\mathrm{~m}, 1 \mathrm{H}), 5.12-5.03(\mathrm{~m}, 2 \mathrm{H}), 4.42$ (s, $2 \mathrm{H}), 3.78-3.71(\mathrm{~m}, 1 \mathrm{H}), 3.18-3.11(\mathrm{~m}, 3 \mathrm{H}), 2.65-2.63$ (m, 2 H), 2.05 (s, $3 \mathrm{H}), 1.86-1.81(\mathrm{~m}, 1 \mathrm{H}), 1.49-1.42$ (m, $1 \mathrm{H}), 1.18-1.11(\mathrm{~m}, 1 \mathrm{H}), 0.91$ (d, J = 7.8 Hz, $6 \mathrm{H})$; ${ }^{13} \mathrm{C} \mathrm{NMR}\left(75 \mathrm{MHz}, \mathrm{CDCl}_{3}\right): \delta 162.4(\mathrm{C}), 137.7(\mathrm{C}), 136.7(\mathrm{CH}), 128.7(2 \times \mathrm{CH}), 127.4(\mathrm{CH}), 126.8(2 \times$ 
$\mathrm{CH}), 115.7\left(\mathrm{CH}_{2}\right), 66.1(\mathrm{CH}), 65.2(\mathrm{CH}), 52.4\left(\mathrm{CH}_{2}\right), 51.3\left(\mathrm{CH}_{2}\right), 48.7\left(\mathrm{CH}_{2}\right), 46.8\left(\mathrm{CH}_{2}\right), 24.4(\mathrm{CH})$, $23.1\left(\mathrm{CH}_{3}\right), 22.4\left(\mathrm{CH}_{3}\right), 14.5\left(\mathrm{CH}_{3}\right)$; IR (neat): 3300, 3064, 3029, 2954, 2868, 2360, 2342, 1652, 1616, $1455 \mathrm{~cm}^{-1} ; R_{f}=0.33(\mathrm{MeOH})$; Anal. Calcd for $\mathrm{C}_{19} \mathrm{H}_{29} \mathrm{~N}_{3}: \mathrm{C}, 76.21 ; \mathrm{H}, 9.76 ; \mathrm{N}, 14.03$. Found: C, 76.38; $\mathrm{H}, 9.96 ; \mathrm{N}, 13.86$.

(4R,5R)-5-(Allylaminomethyl)-1-benzyl-4-isobutyl-2-(methoxymethyl)-4,5-dihydro-1H-imidazole (2e): Colorless oil. $[\alpha]_{\mathrm{D}}^{25}=+13.5\left(c 1.11, \mathrm{CHCl}_{3}\right) ;{ }^{1} \mathrm{H} \mathrm{NMR}\left(300 \mathrm{MHz}, \mathrm{CDCl}_{3}\right): \delta 7.35-7.21(\mathrm{~m}, 5 \mathrm{H})$, 5.83-5.70 (m, $1 \mathrm{H}), 5.11-5.02(\mathrm{~m}, 2 \mathrm{H}), 4.48$ (AB syst., $J=16.0 \mathrm{~Hz}, 2 \mathrm{H}), 4.15$ (AB syst., $J=12.5 \mathrm{~Hz}, 2$ H),3.84-3.77 (m, $1 \mathrm{H}), 3.39$ (s, $3 \mathrm{H}), 3.19-3.13$ (m, $1 \mathrm{H}), 3.09$ (d, J = 6.0 Hz, $2 \mathrm{H}), 2.58$ (d, J = 4.6 Hz, 2 $\mathrm{H}), 1.88-1.74(\mathrm{~m}, 1 \mathrm{H}), 1.50-1.41(\mathrm{~m}, 1 \mathrm{H}), 1.17-1.08(\mathrm{~m}, 1 \mathrm{H}), 0.90(\mathrm{~d}, J=6.7 \mathrm{~Hz}, 6 \mathrm{H}) ;{ }^{13} \mathrm{C} \mathrm{NMR}(75$ $\left.\mathrm{MHz}, \mathrm{CDCl}_{3}\right): \delta 160.9(\mathrm{C}), 138.0(\mathrm{C}), 136.7(\mathrm{CH}), 128.5,127.1(5 \times \mathrm{CH}), 115.5\left(\mathrm{CH}_{2}\right), 67.9\left(\mathrm{CH}_{2}\right), 66.4$ $(\mathrm{CH}), 66.2(\mathrm{CH}), 58.3\left(\mathrm{CH}_{3}\right), 53.3\left(\mathrm{CH}_{2}\right), 51.1\left(\mathrm{CH}_{2}\right), 48.5\left(\mathrm{CH}_{2}\right), 46.7\left(\mathrm{CH}_{2}\right), 24.3(\mathrm{CH}), 22.9\left(\mathrm{CH}_{3}\right)$, $22.5\left(\mathrm{CH}_{3}\right)$; IR (neat): 3300, 3028, 2864, 2361, 2342, 1616, $1455 \mathrm{~cm}^{-1} ; R_{f}=0.31(\mathrm{MeOH})$; Anal. Calcd for $\mathrm{C}_{20} \mathrm{H}_{31} \mathrm{~N}_{3} \mathrm{O}: \mathrm{C}, 72.91 ; \mathrm{H}, 9.48 ; \mathrm{N}, 12.75$. Found: C, 73.09; H, 9.66; N, 12.56 .

(4R,5R)-1,4-Dibenzyl-5-(cyclohexylaminomethyl)-2-(methoxymethyl)-4,5-dihydro-1H-imidazole (2f): Colorless oil. $[\alpha]_{\mathrm{D}}^{25}=+9.5\left(c 0.37, \mathrm{CHCl}_{3}\right) ;{ }^{1} \mathrm{H} \mathrm{NMR}\left(300 \mathrm{MHz}, \mathrm{CDCl}_{3}\right): \delta 7.30-7.06(\mathrm{~m}, 10 \mathrm{H})$, 4.39 (AB syst., $J=16.2 \mathrm{~Hz}, 2 \mathrm{H}$ ), 4.20 (AB syst., $J=12.8 \mathrm{~Hz}, 2 \mathrm{H}), 4.05-3.99$ (m, $1 \mathrm{H}), 3.38$ (s, $3 \mathrm{H}$ ), $3.25(\mathrm{dd}, J=11.4,5.1 \mathrm{~Hz}, 1 \mathrm{H}), 3.07$ (dd, $J=13.4,4.8 \mathrm{~Hz}, 1 \mathrm{H}), 2.50$ (dd, $J=13.4,9.1 \mathrm{~Hz}, 1 \mathrm{H}), 2.36$ $(\mathrm{d}, J=4.8 \mathrm{~Hz}, 1 \mathrm{H}), 2.03-1.94(\mathrm{~m}, 1 \mathrm{H}), 1.57-1.48(\mathrm{~m}, 6 \mathrm{H}), 1.17-1.01(\mathrm{~m}, 3 \mathrm{H}), 0.87-0.72(\mathrm{~m}, 3 \mathrm{H}) ;{ }^{13} \mathrm{C}$ NMR (75 MHz, $\left.\mathrm{CDCl}_{3}\right): \delta 161.2(\mathrm{C}), 137.9$ (C), $137.8(\mathrm{C}), 129.4,128.4,128.1,127.1,127.0,126.0(10$ $\times \mathrm{CH}), 70.0(\mathrm{CH}), 67.8\left(\mathrm{CH}_{2}\right), 63.8\left(\mathrm{CH}_{3}\right), 58.2(\mathrm{CH}), 56.1(\mathrm{CH}), 48.6\left(\mathrm{CH}_{2}\right), 48.1\left(\mathrm{CH}_{2}\right), 42.3\left(\mathrm{CH}_{2}\right)$, $33.1\left(\mathrm{CH}_{2}\right), 33.0\left(\mathrm{CH}_{2}\right), 25.9\left(\mathrm{CH}_{2}\right), 24.6\left(2 \times \mathrm{CH}_{2}\right)$; IR (neat): 3406, 3027, 2926, 2852, 2360, 2342, 1670, 1616, $1451 \mathrm{~cm}^{-1} ; R_{f}=0.32(\mathrm{MeOH})$, Anal. Calcd for $\mathrm{C}_{26} \mathrm{H}_{35} \mathrm{~N}_{3} \mathrm{O}: \mathrm{C}, 77.00 ; \mathrm{H}, 8.80 ; \mathrm{N}, 10.36$. Found: C, 77.21; H, 8.61; N, 10.01 .

(4R,5R)-1,4-Dibenzyl-5-(benzylaminomethyl)-2-methyl-4,5-dihydro-1H-imidazole (2g): Colorless oil. $[\alpha]_{\mathrm{D}}^{25}=-10.9\left(c 0.11, \mathrm{CHCl}_{3}\right) ;{ }^{1} \mathrm{H}$ NMR $\left(300 \mathrm{MHz}, \mathrm{CDCl}_{3}\right): \delta 7.25-7.02(\mathrm{~m}, 15 \mathrm{H}), 4.30(\mathrm{AB}$ syst., $J$ $=16.2 \mathrm{~Hz}, 2 \mathrm{H}), 4.04-4.01(\mathrm{~m}, 1 \mathrm{H}), 3.50(\mathrm{~s}, 2 \mathrm{H}), 3.27(\mathrm{dd}, J=11.4,4.8 \mathrm{~Hz}, 1 \mathrm{H}), 3.07$ (dd, $J=13.6$, $8.7 \mathrm{~Hz}, 1 \mathrm{H}), 2.55(\mathrm{dd}, J=13.6,8.7 \mathrm{~Hz}, 1 \mathrm{H}), 2.38(\mathrm{ddd}, J=16.3,12.2,4.3 \mathrm{~Hz}, 2 \mathrm{H}), 2.01(\mathrm{~s}, 3 \mathrm{H}) ;{ }^{13} \mathrm{C}$ NMR (75 MHz, $\mathrm{CDCl}_{3}$ ): $\delta 162.5$ (C), 140.1 (C), 137.9 (C), 137.6 (C), 129.5, 128.6, 128.4, 128.2, 128.1, 127.8, 127.2, 126.7, $126.0(15 \times \mathrm{CH}), 68.9(\mathrm{CH}), 63.5(\mathrm{CH}), 53.5\left(\mathrm{CH}_{2}\right), 51.0\left(\mathrm{CH}_{2}\right), 48.3\left(\mathrm{CH}_{2}\right), 42.4$ $\left(\mathrm{CH}_{2}\right), 14.5\left(\mathrm{CH}_{3}\right)$; MS (70 eV, EI) m/z (\%) 383 [M+, 4], 264 (25), 263 (93), 173 (88), 91 (100); HRMS (70 eV) calc. for $\mathrm{C}_{26} \mathrm{H}_{29} \mathrm{~N}_{3}\left[M^{+}\right]$383.22356, found 383.2362; IR (neat): 3312, 3061, 3027, 2923, 2360, 2342, 1615, 1453, $1241 \mathrm{~cm}^{-1} ; R_{f}=0.38(\mathrm{MeOH})$; Anal. Calcd for $\mathrm{C}_{26} \mathrm{H}_{29} \mathrm{~N}_{3}: \mathrm{C}, 81.42 ; \mathrm{H}, 7.62 ; \mathrm{N}, 10.96$. Found: C, 81.61; H, 7.45; N, 10.83 .

(4R,5R)-1,4-Dibenzyl-5-(benzylaminomethyl)-2-isopropyl-4,5-dihydro-1H-imidazole

(2h): Colorless oil. $[\alpha]_{\mathrm{D}}^{25}=-19.3\left(c, 1.10 \mathrm{CHCl}_{3}\right) ;{ }^{1} \mathrm{H}$ NMR $\left(300 \mathrm{MHz}, \mathrm{CDCl}_{3}\right): \delta 7.31-6.96(\mathrm{~m}, 15 \mathrm{H}), 4.31$ (AB syst., $J=16.3 \mathrm{~Hz}, 2 \mathrm{H}), 4.11-4.05(\mathrm{~m}, 1 \mathrm{H}), 3.57$ (s, $2 \mathrm{H}), 3.57-3.29$ (m, $1 \mathrm{H}), 3.06$ (dd, $J=13.5,4.2$ $\mathrm{Hz}, 1 \mathrm{H}), 2.66(\mathrm{dd}, J=13.5,8.2 \mathrm{~Hz}, 1 \mathrm{H}), 2.59-2.37(\mathrm{~m}, 3 \mathrm{H}), 1.27(\mathrm{~d}, J=6.9 \mathrm{~Hz}, 6 \mathrm{H}) ;{ }^{13} \mathrm{C} \mathrm{NMR}(75$ $\left.\mathrm{MHz}, \mathrm{CDCl}_{3}\right): \delta 169.8(\mathrm{C}), 140.0(\mathrm{C}), 137.6(\mathrm{C}), 137.4(\mathrm{C}), 129.7,128.4,127.9,127.0,126.5,125.9(15$ $\times \mathrm{CH}), 68.3(\mathrm{CH}), 62.4(\mathrm{CH}), 53.3\left(\mathrm{CH}_{2}\right), 50.8\left(\mathrm{CH}_{2}\right), 47.4\left(\mathrm{CH}_{2}\right), 41.8\left(\mathrm{CH}_{2}\right), 26.0(\mathrm{CH}), 20.9\left(\mathrm{CH}_{3}\right)$, 
$20.0\left(\mathrm{CH}_{3}\right)$; IR (neat): 3313, 3063, 3027, 2924, 2360, 1616, $1454 \mathrm{~cm}^{-1} ; R_{f}=0.42(\mathrm{MeOH})$; Anal. Calcd for $\mathrm{C}_{28} \mathrm{H}_{33} \mathrm{~N}_{3}$ : C, 81.71; H, 8.08; N, 10.21. Found: C, 81.94; H, 7.88; N, 10.06 .

(4R,5R)-1,4-Dibenzyl-5-(benzylaminomethyl)-2-phenyl-4,5-dihydro-1H-imidazole (2i): Colorless oil. $[\alpha]_{\mathrm{D}}^{25}=-43.3\left(c 0.21, \mathrm{CHCl}_{3}\right) ;{ }^{1} \mathrm{H} \mathrm{NMR}\left(200 \mathrm{MHz}, \mathrm{CDCl}_{3}\right): \delta 7.72-7.04(\mathrm{~m}, 20 \mathrm{H}), 4.21(\mathrm{~s}, 2 \mathrm{H})$, 4.19-4.11 (m, 1 H), 3.59 (s, 2 H), 3.35-3.29 (m, 1 H), 3.04 (dd, $J=13.3,4.6$ Hz, 1 H), 2.51-2.39 (m, 3 $\mathrm{H}) ;{ }^{13} \mathrm{C}$ NMR $\left(75 \mathrm{MHz}, \mathrm{CDCl}_{3}\right): \delta 165.5(\mathrm{C}), 140.2(\mathrm{C}), 137.8,137.7(3 \times \mathrm{C}), 129.9,129.6,128.6,128.5$, 128.4, 128.1, 127.8, 127.7, 127.4, 126.7, $126.1(20 \times \mathrm{CH}), 70.3(\mathrm{CH}), 63.1(\mathrm{CH}), 53.5\left(\mathrm{CH}_{2}\right), 52.1$ $\left(\mathrm{CH}_{2}\right), 51.0\left(\mathrm{CH}_{2}\right), 42.2\left(\mathrm{CH}_{2}\right)$; MS (70 eV, EI) m/z (\%) $445\left[M^{+}, 1\right], 326(15), 325(58), 235(62), 91$ (100), 65 (8); HRMS (70 eV) calc. for $\mathrm{C}_{31} \mathrm{H}_{31} \mathrm{~N}_{3}\left[M^{+}\right]$445.2513, found 445.2520; IR (neat): 3313, 3061, 3027, 2921, 2360, 2342, 1605, 1595, 1495, $1453 \mathrm{~cm}^{-1} ; R_{f}=0.45(\mathrm{MeOH})$; Anal. Calcd for $\mathrm{C}_{31} \mathrm{H}_{31} \mathrm{~N}_{3}: \mathrm{C}_{\text {, }}$ 83.56; H, 7.01; N, 9.43. Found: C, 83.71; H, 7.10; N, 9.32.

(4R,5R)-1-Benzyl-5-(benzylaminomethyl)-4-(benzyloxymethyl)-2-(methoxymethyl)-4,5-dihydro$1 \boldsymbol{H}$-imidazole (2j): Colorless oil. $[\alpha]_{\mathrm{D}}^{25}=+4.3\left(c 1.20, \mathrm{CHCl}_{3}\right) ;{ }^{1} \mathrm{H}$ NMR $\left(300 \mathrm{MHz}, \mathrm{CDCl}_{3}\right): \delta 7.31$ 7.19 (m, 15 H), 4.51-4.42 (m, 4 H), 4.16-4.03 (m, 3 H), 3.80-3.51 (m, 3 H), 3.47-3.43 (m, 2 H), 3.40 (s, 3 $\mathrm{H}), 2.67$ (d, $J=4.6 \mathrm{~Hz}, 2 \mathrm{H}) ;{ }^{13} \mathrm{C}$ NMR $\left(75 \mathrm{MHz}, \mathrm{CDCl}_{3}\right): \delta 162.4(\mathrm{C}), 140.2(\mathrm{C}), 138.1(\mathrm{C}), 137.7$ (C), 128.5, 128.1, 127.8, 127.6, 127.3, 127.0, $126.7(15 \times \mathrm{CH}), 73.1\left(\mathrm{CH}_{2}\right), 72.8\left(\mathrm{CH}_{2}\right), 68.6(\mathrm{CH}), 67.8$ $\left(\mathrm{CH}_{2}\right), 63.2(\mathrm{CH}), 58.3\left(\mathrm{CH}_{3}\right), 53.7\left(\mathrm{CH}_{2}\right), 51.0\left(\mathrm{CH}_{2}\right), 48.1\left(\mathrm{CH}_{2}\right)$; MS (70 eV, EI) $\mathrm{m} / z(\%) 443\left[M^{+}, 6\right]$, 323 (36), 233 (51), 203 (89), 171 (47), 91 (100); HRMS (70 eV) calc. for $\mathrm{C}_{28} \mathrm{H}_{33} \mathrm{~N}_{3} \mathrm{O}_{2}\left[M^{+}\right]$443.2567, found 443.2567; IR (neat): 3406, 3324, 3028, 2926, 2857, 1674, 1520, 1496, 1454, $1113 \mathrm{~cm}^{-1} ; R_{f}=0.42$ $(\mathrm{MeOH})$; Anal. Calcd for $\mathrm{C}_{28} \mathrm{H}_{33} \mathrm{~N}_{3} \mathrm{O}_{2}:$ C, 75.81; H, 7.50; N, 9.47. Found: C, 75.75; H, 7.65; N, 9.38.

(4R,5R)-1-Benzyl-5-(benzylaminomethyl)-4-(benzyloxymethyl)-2-phenyl-4,5-dihydro-1H-

imidazole (2k): Colorless oil. $[\alpha]_{\mathrm{D}}^{25}=-18.0\left(c 0.10, \mathrm{CHCl}_{3}\right) ;{ }^{1} \mathrm{H} \mathrm{NMR}\left(200 \mathrm{MHz}, \mathrm{CDCl}_{3}\right): \delta 7.65-7.16$ $(\mathrm{m}, 20 \mathrm{H}), 4.52-4.37(\mathrm{~m}, 4 \mathrm{H}), 4.15-4.11(\mathrm{~m}, 1 \mathrm{H}), 3.89-3.52(\mathrm{~m}, 5 \mathrm{H}), 3.36(\mathrm{dd}, J=9.2,6.9 \mathrm{~Hz}, 1 \mathrm{H})$, 2.78-2.73 (m, $2 \mathrm{H}) ;{ }^{13} \mathrm{C}$ NMR (75 MHz, $\mathrm{CDCl}_{3}$ ): $\delta 166.7$ (C), $140.3(\mathrm{C}), 138.7(\mathrm{C}), 137.6(\mathrm{C}), 131.1(\mathrm{C})$, 130.0, 128.2, 127.9, 127.6, 127.5, 127.4, 127.3, $126.8(20 \times \mathrm{CH}), 73.2\left(\mathrm{CH}_{2}\right), 73.0\left(\mathrm{CH}_{2}\right), 69.2(\mathrm{CH})$, $63.5(\mathrm{CH}), 53.8\left(\mathrm{CH}_{2}\right), 52.0\left(\mathrm{CH}_{2}\right), 51.0\left(\mathrm{CH}_{2}\right)$; MS (70 eV, EI) $\mathrm{m} / z(\%) 475\left[M^{+}, 3\right], 355(27), 325(40)$, 248 (34), 235 (76), 91 (100); HRMS (70 eV) calc. for $\mathrm{C}_{32} \mathrm{H}_{33} \mathrm{~N}_{3} \mathrm{O}\left[M^{+}\right]$475.2618, found 475.2609; IR (neat): 3311, 3062, 3027, 2856, 1675, 1615, 1595, 1496, $1454 \mathrm{~cm}^{-1} ; R_{f}=0.42(\mathrm{MeOH})$; Anal. Calcd for $\mathrm{C}_{32} \mathrm{H}_{33} \mathrm{~N}_{3} \mathrm{O}: \mathrm{C}, 80.81 ; \mathrm{H}, 6.99 ; \mathrm{N}, 8.83$. Found: C, 81.07; H, 7.05; N, 8.69. 
'Bu,

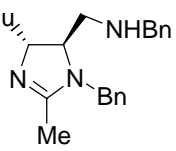

2a
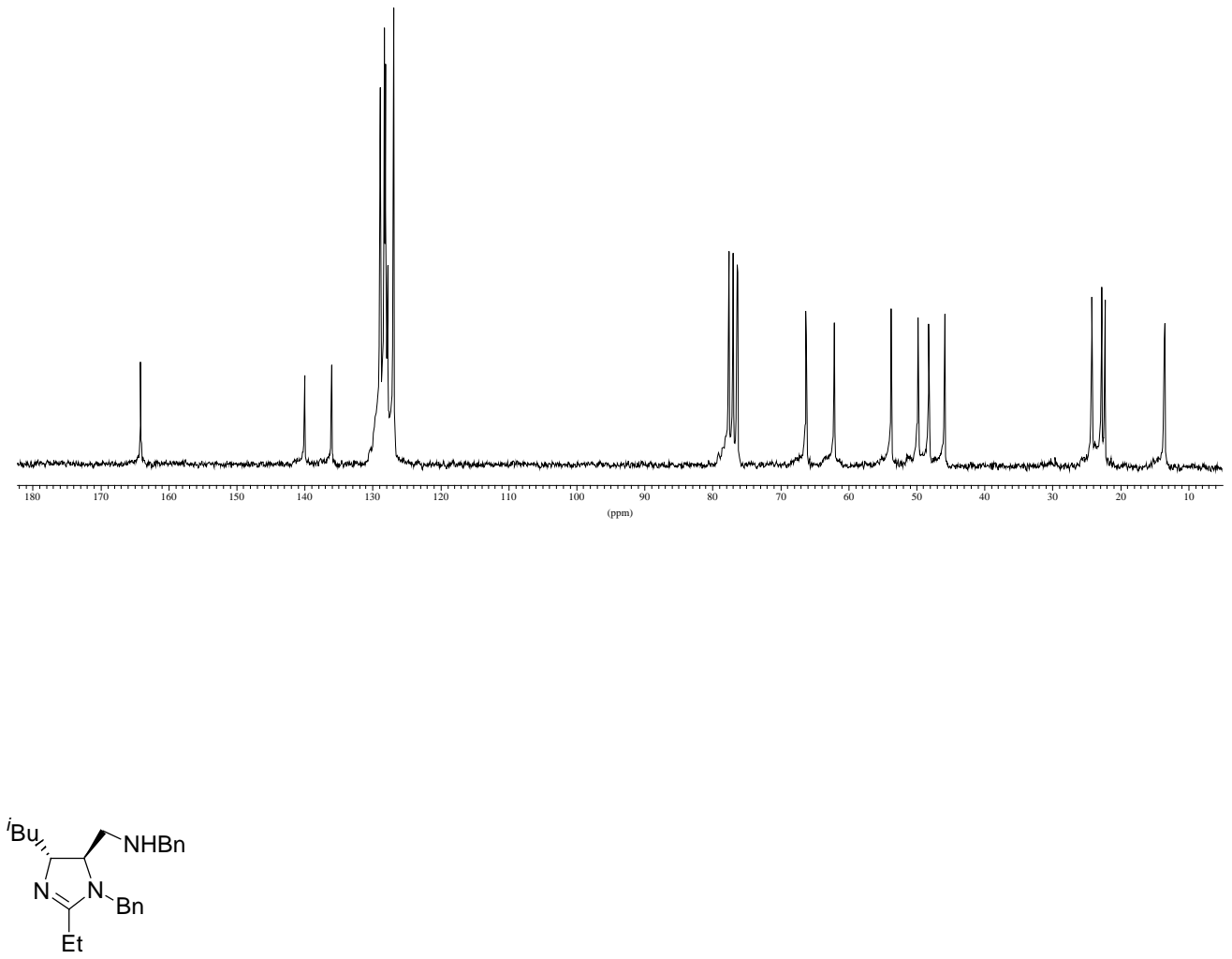

$2 b$

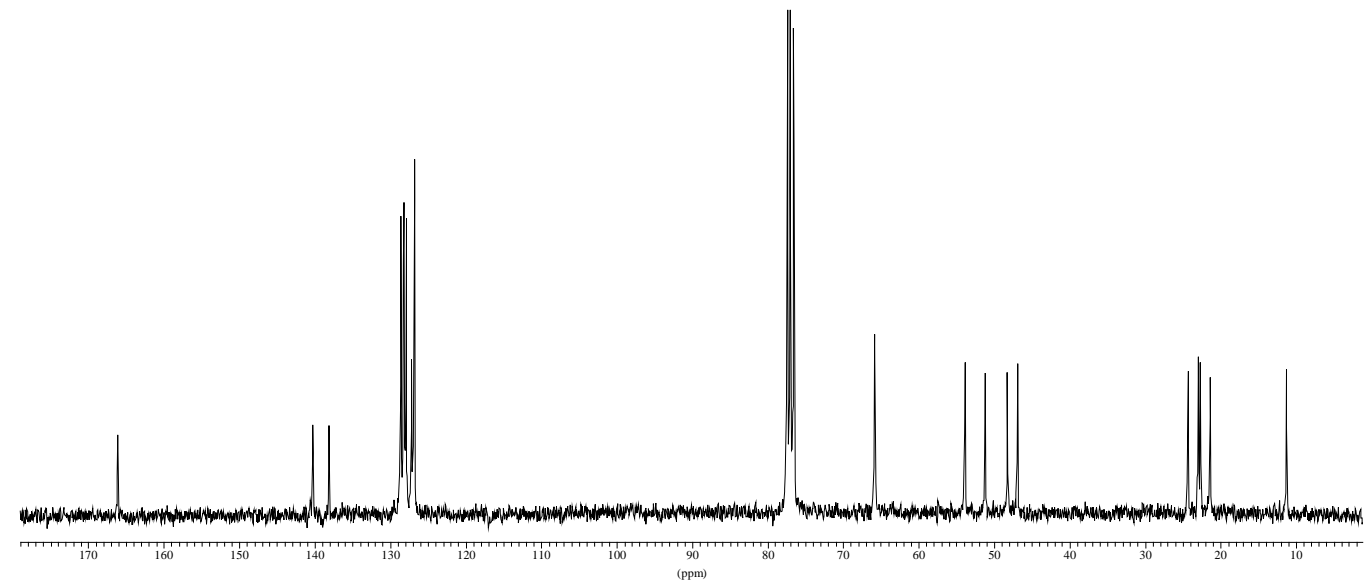


'Bu,<smiles>CCCC1=N[C@@H](C)C(CNCc2ccccc2)N1Cc1ccccc1</smiles>

2c

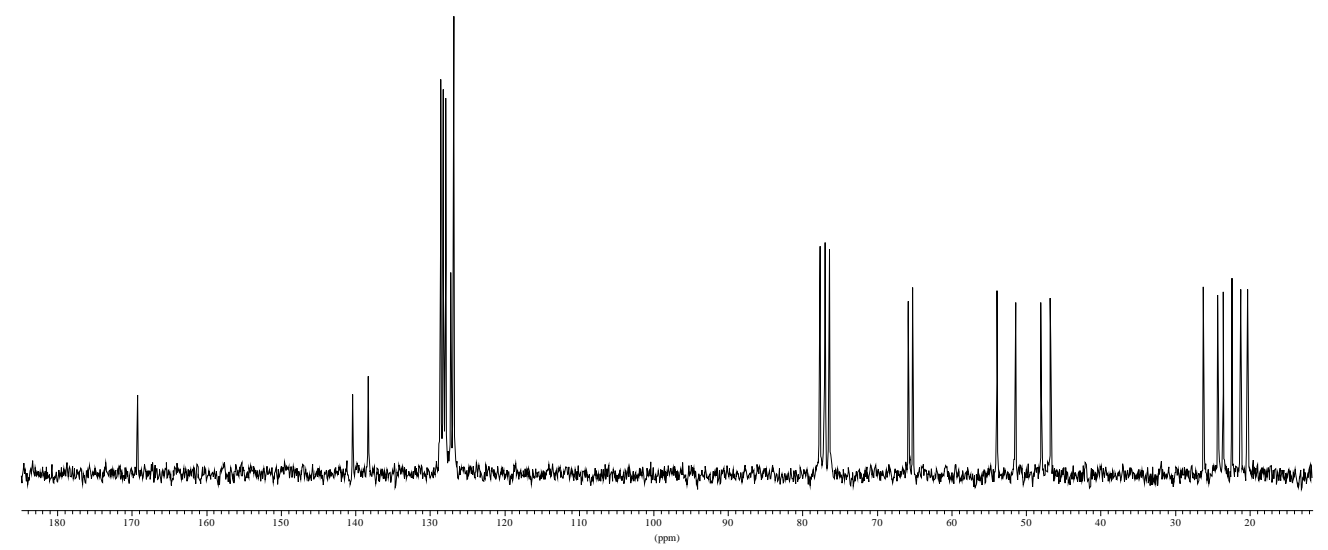

${ }_{\mathrm{Me}}^{\mathrm{B} u}$

2d

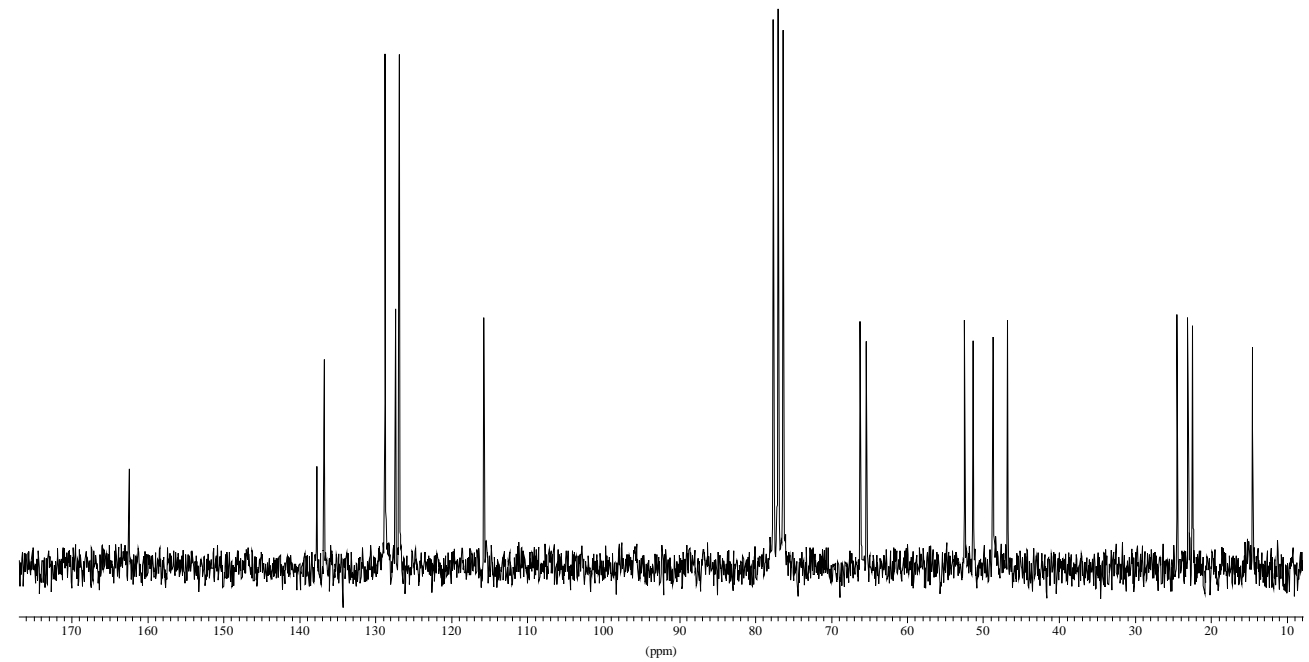


(c)

$2 e$
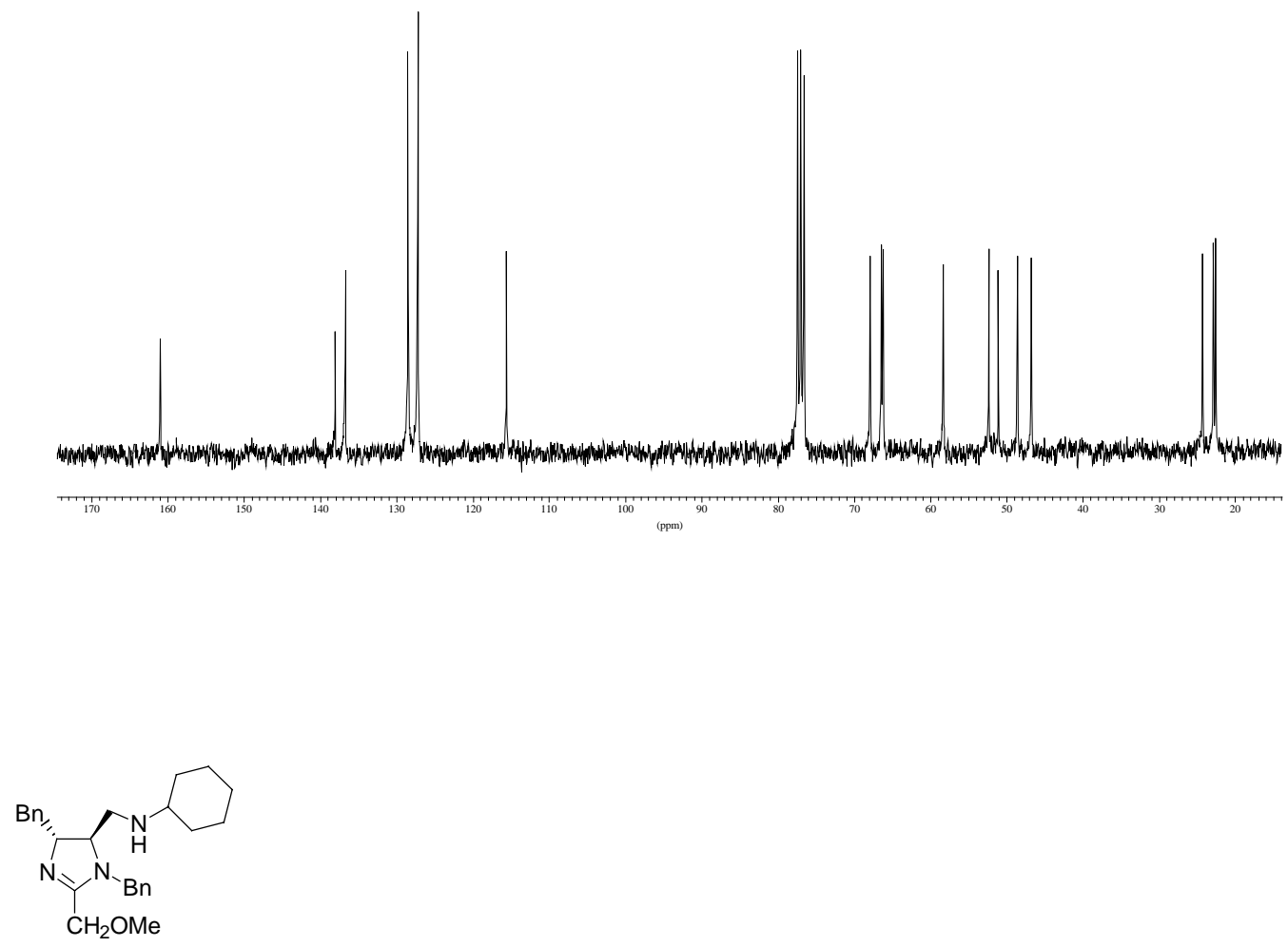

2f

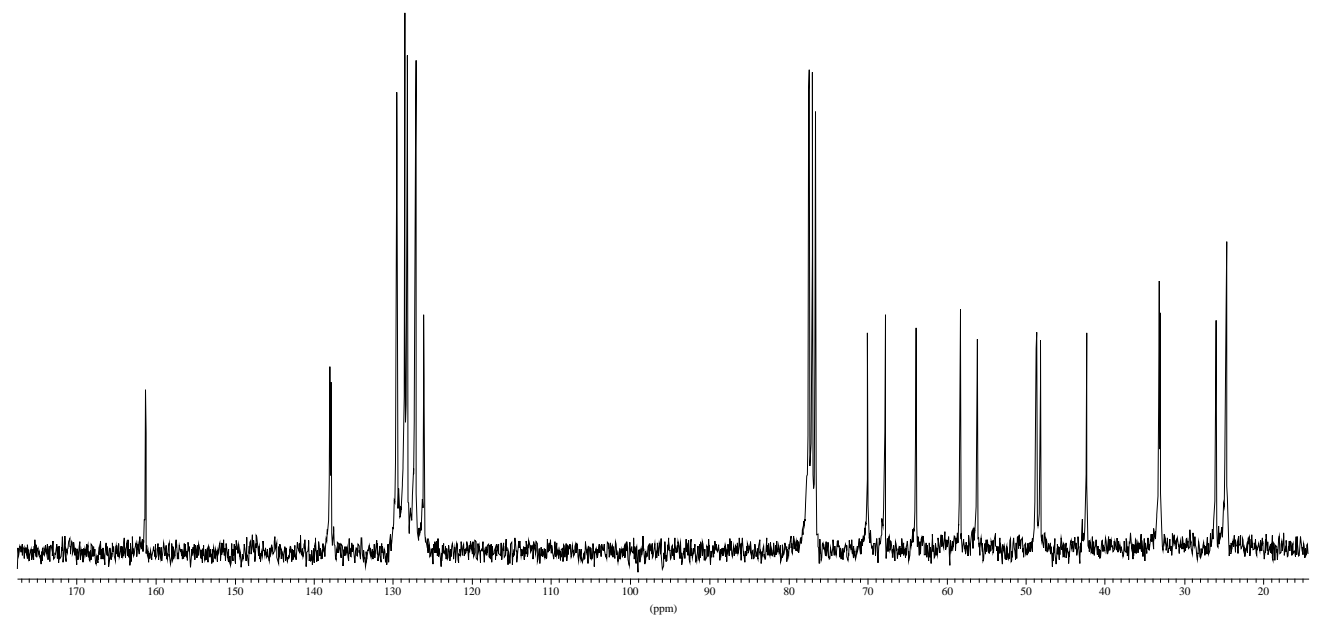



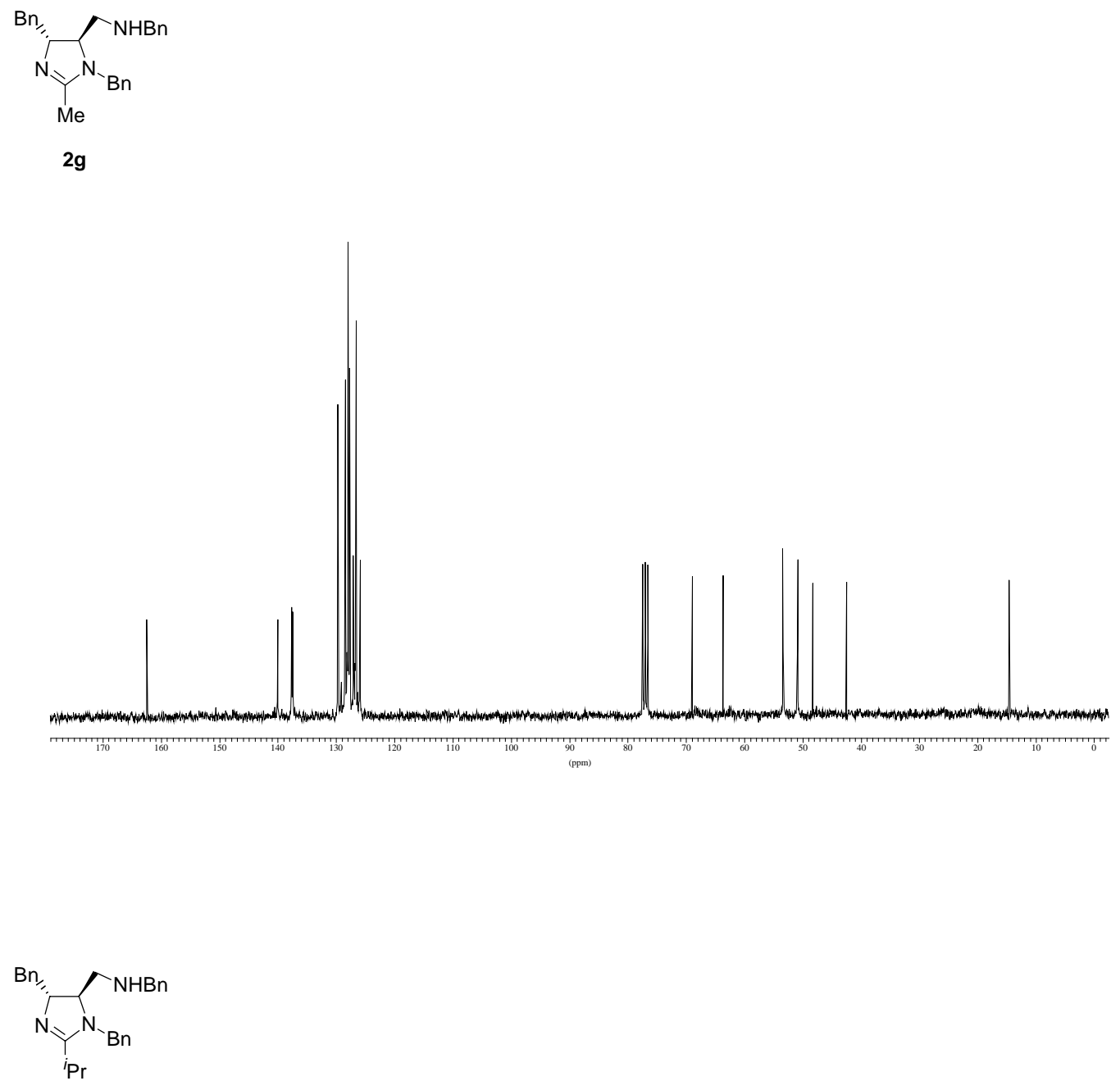

2h

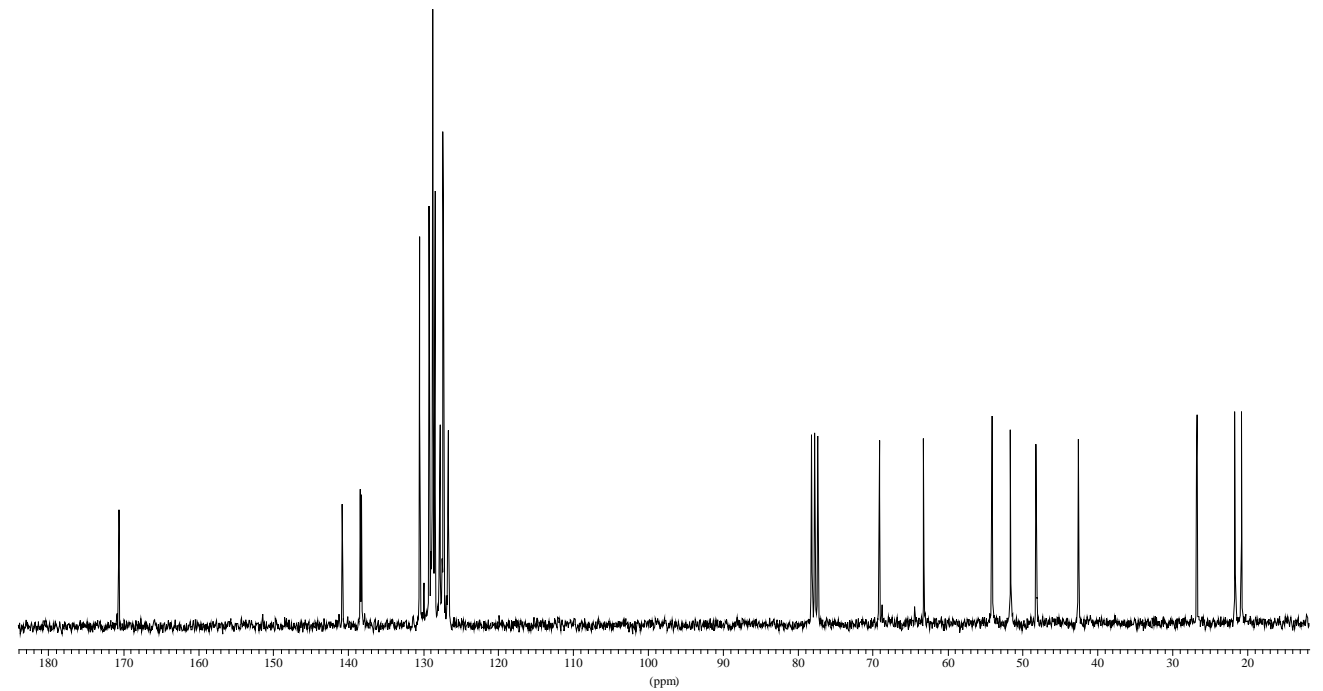




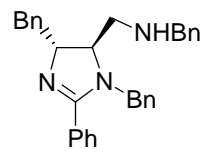

2i
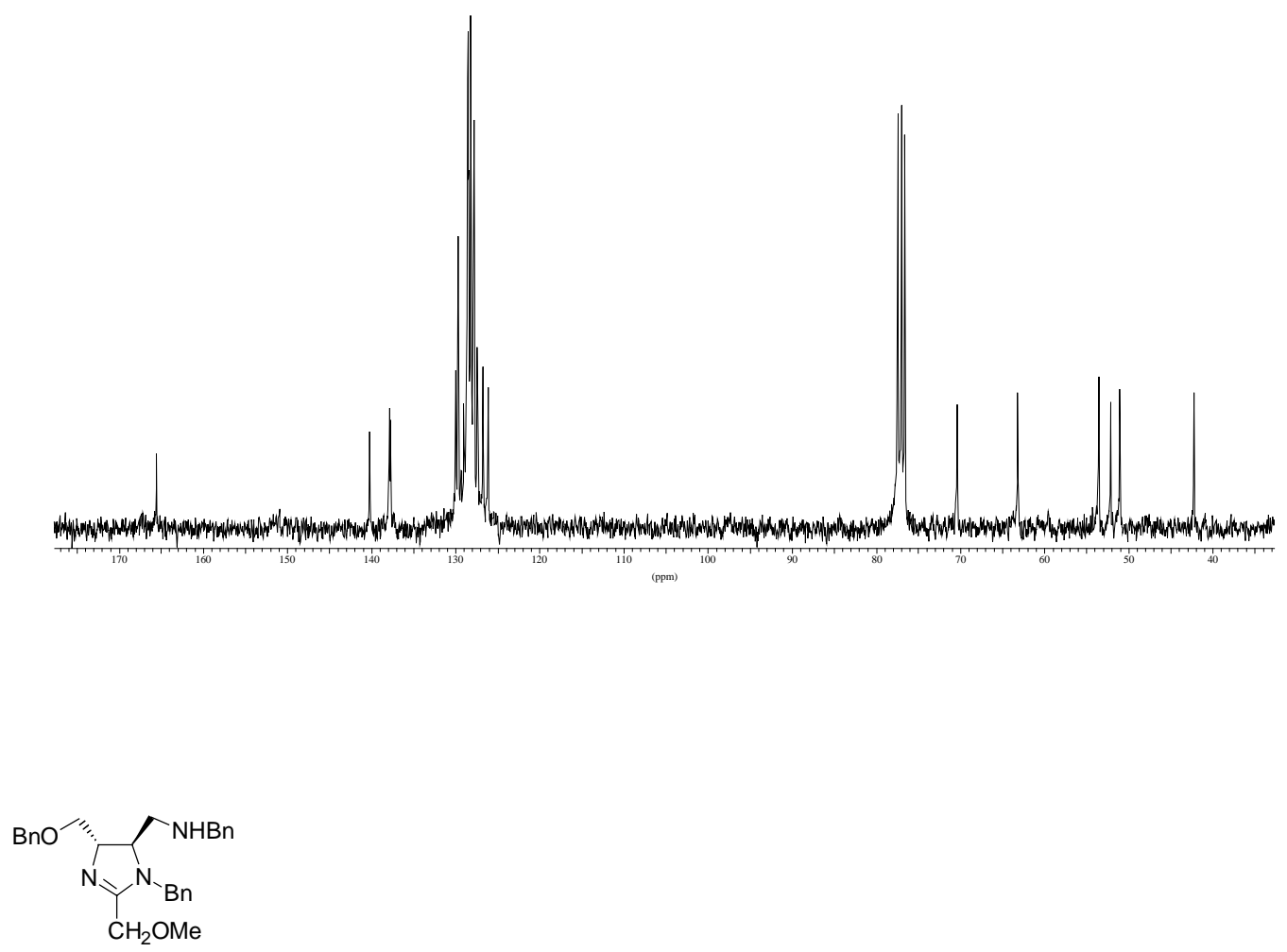

2j

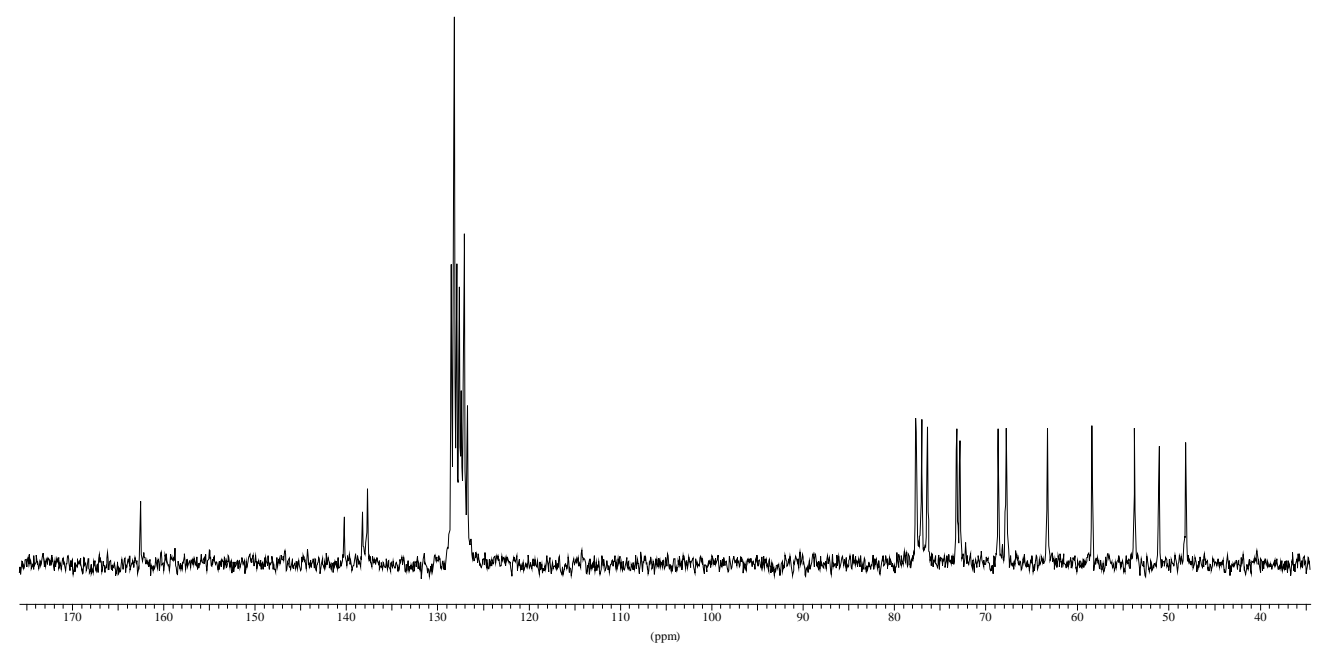




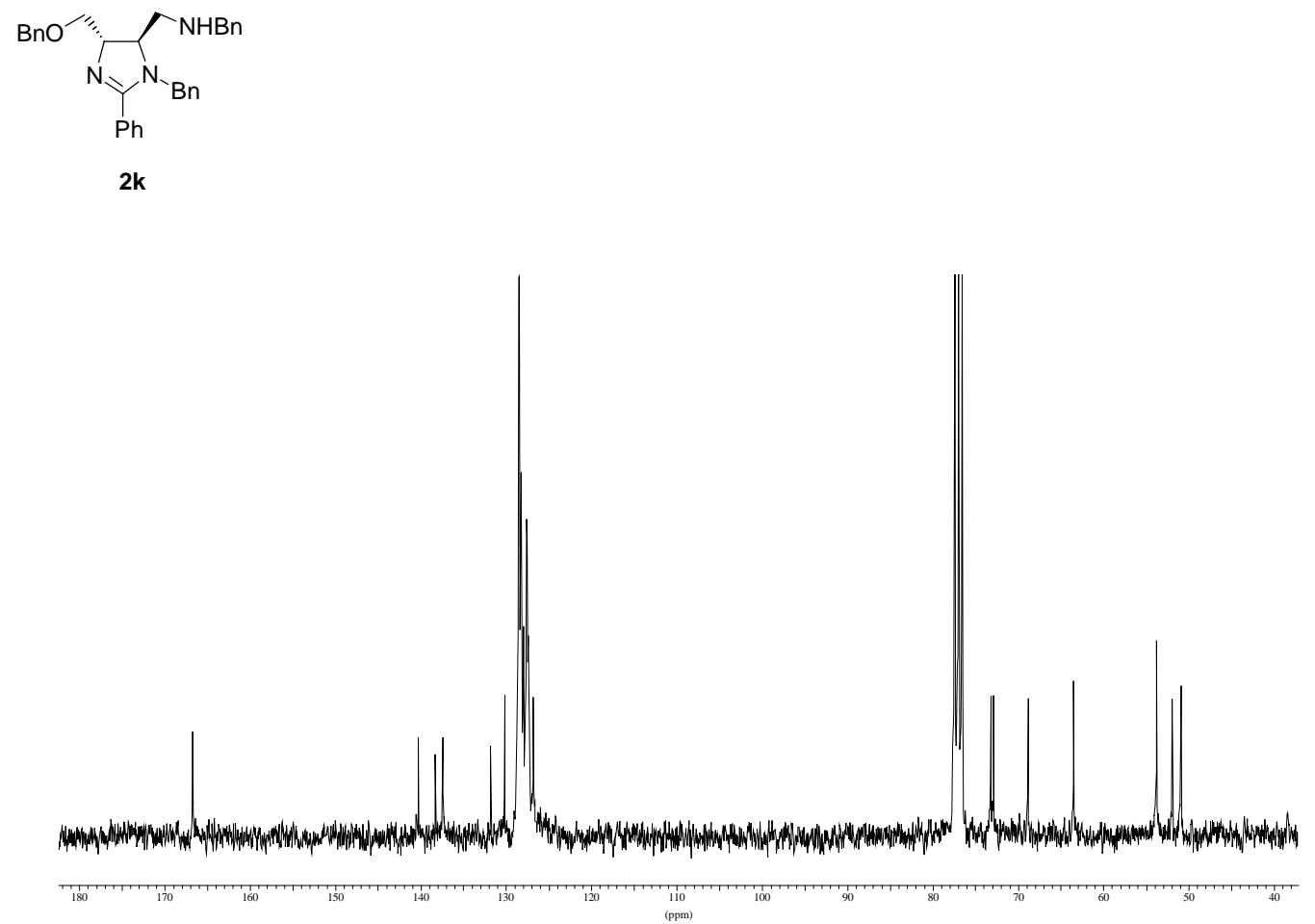

NOESY OF :

'Bu,

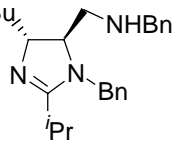

2c 


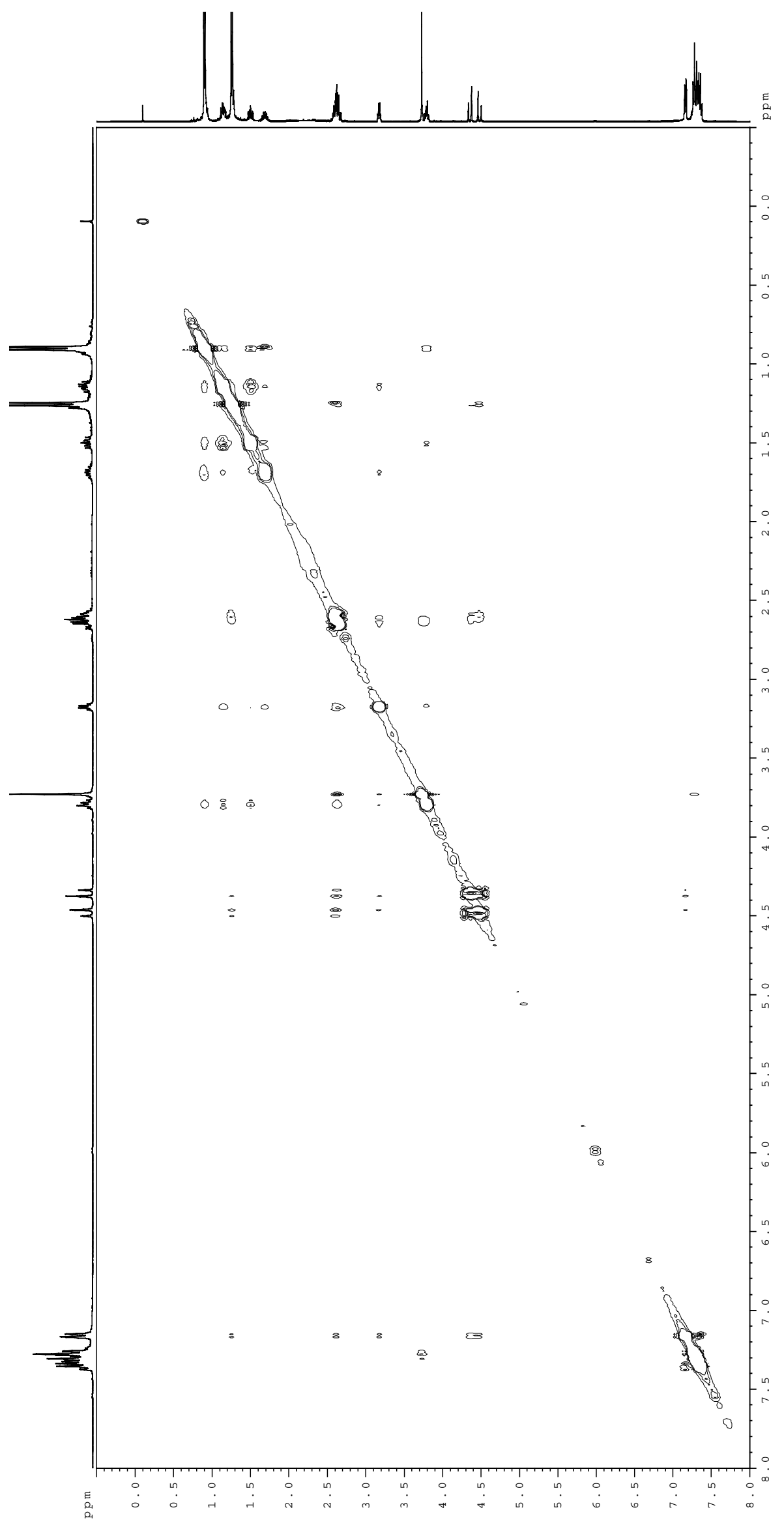




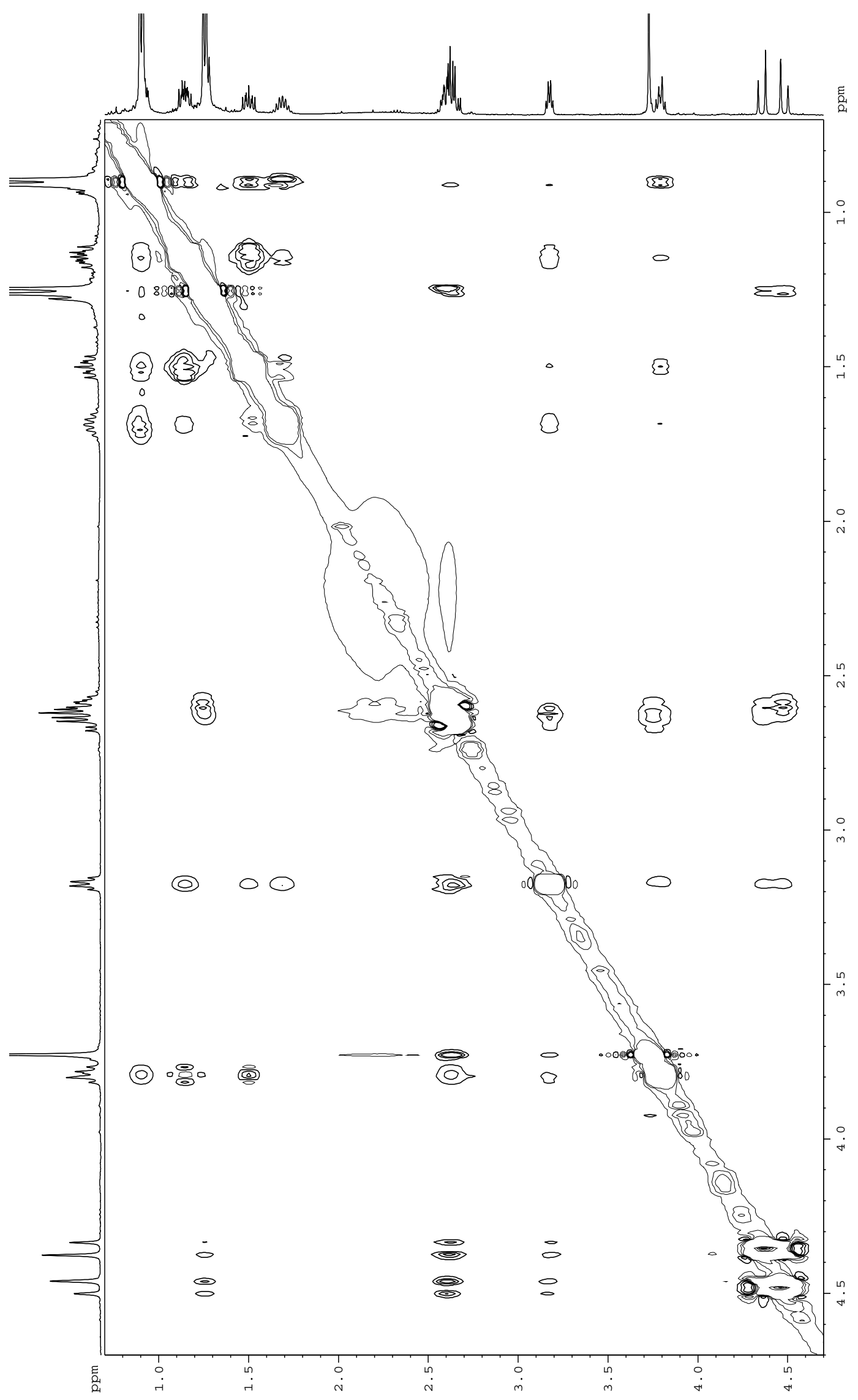

I 